\title{
MEJORAMIENTO DE PROPIEDADES MECÁNICAS DEL PAPEL PLEGADIZA RECUPERADO DE RESIDUOS SÓLIDOS MUNICIPALES
}

\author{
IMPROVEMENT OF RECOVERY FOLDING PAPER \\ MECHANICAL PROPERTIES FROM MUNICIPAL SOLID \\ WASTE
}

\author{
Johnatan Alberto Gutiérrez-R. ${ }^{1}$, Luis Fernando Marmolejo-Rebellón ${ }^{2}$, Fred Albán Achinte ${ }^{3}$, Wilmar Alexander Torres ${ }^{4}$, \\ Patricia Torres-Lozada ${ }^{5}$
}

\begin{abstract}
${ }^{1}$ Ing. Sanitario, Est. M.Sc Ingeniería Sanitaria y Ambiental, Universidad del Valle, Cali, Colombia, e-mail: johnatan.gutierrez@ correounivalle.edu.co; ${ }^{2}$ Ing. Sanitario. M.Sc., Ph.D. Prof. asistente, Facultad de Ingeniería, Universidad del Valle, Cali, Colombia, e-mail: luis.marmolejo@correounivalle.edu.co; ${ }^{3}$ Ing. de Materiales, M.Sc., Prof. Asociado, Facultad de Ingeniería, Universidad del Valle, Cali, Colombia, e-mail: fred.alban@correounivalle.edu.co; ${ }^{4}$ Estadístico, Prof. Auxiliar, Facultad de Ingeniería, Universidad del Valle, e-mail: alexandertor@gmail.com; ${ }^{5}$ Ing. Sanitaria, M.Sc., Ph.D. Prof. Titular, Facultad de Ingeniería, Universidad del Valle, Cali, Colombia, e-mail: patricia.torres@correounivalle.edu.co, Dirección correspondencia: Carrera. 100 No. 13-00 Edificio. 336 - Oficina 2016. Cali, Valle del Cauca - Colombia, e-mail: luis.marmolejo@correounivalle.edu.co
\end{abstract}

Rev. U.D.C.A Act. \& Div. Cient. 17(1): 237-243, Enero-Junio, 2014

\section{RESUMEN}

Entre los residuos sólidos municipales - RSM, el papel es uno de los materiales con mayor potencial de aprovechamiento; sin embargo, el papel plegadiza (PL) recuperado en las plantas de manejo de residuos sólidos municipales - PMRS, de los pequeños municipios del Valle del Cauca, Colombia, presenta poca posibilidad de comercialización, haciendo necesario buscar opciones de aprovechamiento, priorizando en alternativas in situ. En este artículo, se evaluó el efecto de la mezcla de Papel Bond (PB) con Papel Plegadiza blanco y café (PLB y PLC) sobre la tensión y la rigidez flexural. Los tratamientos evaluados para cada uno de los dos tipos de PL fueron PL:PB 70:30, 80:20, 90:10 y 100:00. En los tratamientos PLB:PB no se encontraron diferencias significativas en la tensión y en la rigidez flexural; todos los tratamientos con $\mathrm{PB}$ presentaron mayores valores que el tratamiento control (PLB:PB 100:00). Para los tratamientos PLC:PB solamente el 70:30 incidió en la tensión, aumentándola y la rigidez flexural en todos los tratamientos que contenían PB fue menor que la del tratamiento control. En general, los resultados muestran que incorporar PB puede ser una opción para valorizar el PL y las fracciones de las mezclas dependerán de los requerimientos del producto.

Palabras clave: Aprovechamiento de RSM, plantas de aprovechamiento, reciclaje de papel, tensión y rigidez flexural.

\section{SUMMARY}

Among municipal solid waste - MSW, paper is one of the most promising materials for recycling. However, folding paper (PL), the second category of the paper received in Solid Waste Management Facilities of small municipalities of Valle del Cauca - Colombia, has little marketability and is being sent to final disposal. Therefore, it is convenient to identify options to valorize it. For this proposal, in this research the effect of mixing Paper Bond (PB) with white or brown folding paper (PLB and PLC), taking tension and flexural rigidity as response variables was evaluated. The treatments evaluated for each of the two types of PL were PL: PB 70:30, 80:20, 90:10 and 100:00. In PLB:PB treatments, no significant differences in tension between the treatments were found. For the flexural rigidity, all treatments with PB showed a higher value than the control (PLB: PB 100:00). For treatments PLC:PB, only 70:30 showed a higher value of tension than 100:00 treatment. Additionally, the treatments that contain PLC, presented lower values of flexural rigidity comparing with the treatment 100 PLC:OPB. It can be concluded that to incorporate PB may be an option to improve the recovery and valorization of PL.

Key words: Use of RSM, exploitation plants, paper recycling, tension and flexural rigidity. 


\section{INTRODUCCIÓN}

Una de las alternativas para el aprovechamiento de los RSM en Colombia es la implementación de Plantas de Manejo de Residuos Sólidos - PMRS, cuyo objeto fundamental es el aprovechamiento y la valorización de la mayor cantidad posible de residuos y la disposición final de aquellos materiales que no tienen posibilidad de ser aprovechados o no existen opciones para la transformación o mercadeo en el entorno (Marmolejo et al. 2009).

El papel es uno de los materiales contenidos en los RSM que tiene mayor potencial de aprovechamiento y cuyo reprocesamiento demanda menos energía y causa menos emisiones que la fabricación de cantidades equivalentes, a partir de materiales vírgenes (Merrild et al. 2008). Algunas alternativas de aprovechamiento de papel son la obtención de energía por medio de su incineración y elaboración de madera sintética, consistente en una mezcla de productos plásticos (MAVDT \& EPAM, 2008); sin embargo, dado que las PMRS se ubican principalmente en pequeños municipios (SSPD, 2008), en donde la cantidad de material y las limitaciones técnicas y financieras dificultan la aplicación de tecnologías con altos requerimientos de inversión, operación y mantenimiento es conveniente estimular opciones de aprovechamiento in situ, que prioricen el empleo de mano de obra, sobre el uso de tecnologías altamente mecanizadas.

En el caso de los RSM generados en PMRS del Valle del Cauca, Colombia, el papel constituye, aproximadamente, 3\% del total de los RSM, siendo el Papel Bond (PB) y el Papel Plegadiza (PL) las categorías predominantes, mientras que el PB tiene una amplia demanda en el mercado regional; el PL tiene poca posibilidad de comercialización, siendo necesario almacenarlo durante largos períodos de tiempo o enviarlo a disposición.
En el caso del PL existe dos subcategorías que se pueden diferenciar visualmente por el color de su base: blanco (PLB) y café (PLC), cuyas características físicas y mecánicas difieren, debido a las fibras, a partir de las cuales se fabrican; la primera está constituida por pulpa de origen químico, que le otorga unas fibras puras y largas, que se traduce en resistencia y en estabilidad, mientras que la segunda se fabrica a partir de fibras mecánicas, por lo que se presentan fibras impuras y cortas, que le restan resistencia y estabilidad a los papeles producidos (Smook, 1990).

Una de las opciones que podría contribuir a incrementar el potencial de aprovechamiento del PL es mejorar sus propiedades mecánicas, lo cual, se puede lograr mediante la mezcla con otros materiales (Smook, 1990). Entre las propiedades mecánicas a mejorar están la tensión y la rigidez flexural, las que le aportan resistencia a las fibras frente a esfuerzos tensores y plasticidad contra fuerzas flectoras, que le permitan retomar su forma original respectivamente (Casey, 1991). Para tal fin, en este artículo, se evaluó el efecto de la incorporación de Papel Bond (PB) al Papel Plegadiza (PL), en diferentes proporciones, sobre el comportamiento de las variables de respuesta tensión y rigidez flexural.

\section{MATERIALES Y MÉTODOS}

Las muestras de PB fueron obtenidas a partir del papel desechado en oficinas y las de PLB y PLC (Figura 1), a partir de los RSM recibidos en tres PMRS, ubicadas en el Norte del Valle del Cauca, verificando que los materiales estuvieran secos; previo a la conformación de las mezclas, cada uno de los materiales fue homogenizado y para facilitar el procesamiento posterior, se redujo el tamaño de los materiales, picando el PB, en una trituradora de seguridad y fraccionando en guillotina el PL (PLB y PLC), en trozos de 5 x $2 \mathrm{~cm}$.

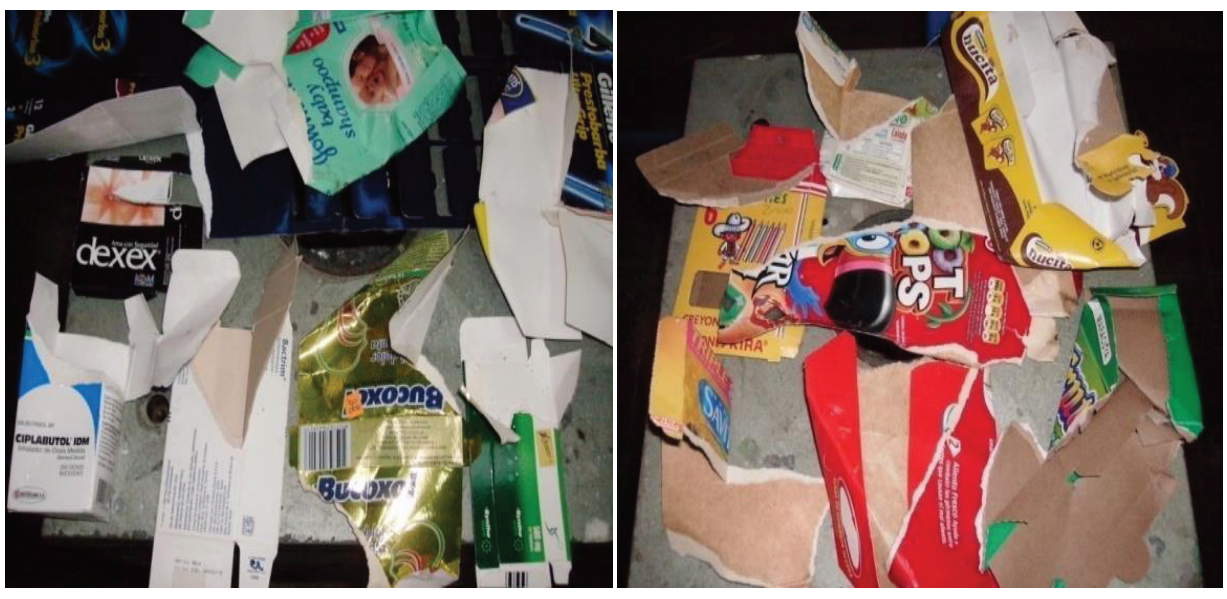

Figura 1. Papel plegadiza blanco y café. 
Montaje experimental: Para cada uno de los tipos de PL (PLB y PLC), se evaluaron los tratamientos mostrados en la tabla 1. La máxima proporción de PB incorporada al PL fue $30 \%$, debido a que se consideró que valores superiores harían poco atractiva la mezcla, debido a la alta demanda del PB.

Tabla 1. Tratamientos para evaluar repulpeo de plegadiza.

\begin{tabular}{|c|c|}
\hline Tratamientos & PL:PB \\
\hline $\mathrm{T}_{i 1}$ (Control) & $100: 0$ \\
\hline $\mathrm{T}_{i 2}$ & $90: 10$ \\
\hline $\mathrm{T}_{i 3}$ & $80: 20$ \\
\hline $\mathrm{T}_{i 4}$ & $70: 30$ \\
\hline
\end{tabular}

$i=1$ (PLB); $i=2$ (PLC).

Por cada color de PL, se tuvieron cuatro tratamientos y se elaboraron tres unidades experimentales (UE) de cada uno; cada UE, se obtuvo del repulpeo de una masa de $843 \mathrm{~g}$ de mezcla de PB y PL y su conformación se hizo adaptando el proceso de elaboración de papel artesanal para la mezcla de papeles, propuesto por Pedraza et al. (2007) e, incluyó, las siguientes etapas: Humectación: a masa de papel de cada UE fue sumergida durante 24 horas en cinco litros de agua, del acueducto municipal. Repulpeo: la masa humectada fue licuada durante cinco minutos en una licuadora industrial de ocho litros de capacidad, con potencia de 0,5 HP y una velocidad de 3500RPM. Una vez obtenida la pulpa, se calculó la cantidad necesaria para elaborar una hoja de $600 \mathrm{~g} / \mathrm{m}^{2} \mathrm{y}$ $16 \mathrm{~cm}$ de diámetro; de cada tratamiento, se obtenían cinco hojas, denominadas unidades observacionales o submuestras. Secado: las hojas formadas, se secaron a temperatura ambiente durante cinco días. El gramaje de las hojas secas, se determinó utilizando la Ecuación 1 (Casey, 1991):

$$
G=\frac{P}{A} \quad \text { Ec. } 1 .
$$

Donde, G: gramaje de la hoja formada $\left(\mathrm{g} / \mathrm{m}^{2}\right)$; P: masa de la hoja formada $(\mathrm{g})$ y A: área de la hoja formada $\left(\mathrm{m}^{2}\right)$.

Ensayos mecánicos: De cada una de las hojas formadas, se obtuvieron dos probetas, una para la prueba de tensión, de $15 \mathrm{~mm}$ de ancho y, la otra, para la de rigidez flexural, de $35 \mathrm{~mm}$ de ancho; el largo era determinado por los bordes de las unidades observacionales, verificando que fuera constante para cada una de las determinaciones.

El ensayo de tensión, se realizó bajo las condiciones de la Norma TAPPI T494 OM-2001 (TAPPI, 2001); para ello, la probeta fue llevada hasta el punto de falla en una máquina Universal JJ Instruments T30K, determinando el esfuerzo en Newtons; la carga aplicada, se incrementó a una velocidad de $2 \mathrm{~mm} / \mathrm{min}$, hasta llegar a la carga de rotura.
Para los ensayos de rigidez flexural, se utilizó el procedimiento propuesto por Casey (1991), aplicando la carga a una velocidad, de $2 \mathrm{~mm} / \mathrm{min}$, sobre la cara superficial de la probeta, hasta llegar a una extensión de $5 \mathrm{~mm}$, sobre el eje horizontal de ésta; para ello, se utilizó un equipo de ensayos universales Tinius Olsen H50KS. La probeta, se apoyaba en los extremos de un soporte con una luz de $123 \mathrm{~mm}$. Con los registros de extensión, se obtenía una curva de Carga-Deformación, en la cual, se encontraba la pendiente $\mathrm{F} / \mathrm{m}$, que se introducía en la Ecuación 2 (Casey, 1991), para obtener el módulo de elasticidad del material. Finalmente, se calculaba la rigidez flexural, de acuerdo con la Ecuación 3 (Casey, 1991):

$$
E_{B}=\frac{L^{3} m}{4 b d^{3}} \quad \text { Ec. } 2
$$

Donde, EB: Módulo de elasticidad $\left(\mathrm{N} / \mathrm{mm}^{2}\right)$; m: relación $\mathrm{F} / \mathrm{m}$ obtenida del ensayo; L: Distancia entre apoyos (mm); b: Ancho de la probeta $(\mathrm{mm})$ y d: Espesor de la probeta $(\mathrm{mm})$.

$$
R=\frac{E T^{3} W}{12 L^{2}} \quad \text { Ec. } 3
$$

Donde, R: Rigidez Flexural (MPa o N/mm²); E: Módulo de elasticidade $E_{в}\left(M P a \circ \mathrm{N} / \mathrm{mm}^{2}\right)$; T: Espesor de la probeta (mm); W: Ancho de la probeta (mm) y L: Distancia entre los apoyos del soporte $(\mathrm{mm})$.

Procesamiento estadístico de los datos: Para evaluar el efecto de la mezcla sobre las variables objeto de estudio, se empleó un diseño de experimentos completamente al azar de dos factores con sub-muestreo (Kuehl, 2001), cuyo modelo estadístico se muestra en la Ecuación 4; este modelo, se empleó, debido a que se tenían varias mediciones por unidad experimental, lo cual, además de la variabilidad existente entre las unidades experimentales, introduce la variabilidad entre las observaciones.

$$
\begin{aligned}
& y_{i j k l}=\mu+\tau_{i}+\gamma_{j}+(\tau \gamma)_{i j}+e_{i j k}+\delta_{i j k l} \\
& i=1,2,3,4 ; j=1,2,3,4 ; k=1,2,3 ; l=1,2,3,4,5
\end{aligned}
$$


Donde, $\gamma_{j j k l}$ : Medición de la variable de respuesta (Tensión, Rigidez); $\mu$ : Media general del modelo; $\tau_{i}$ : Efecto fijo asociado al i-esimo nivel del factor PL; $\gamma_{j}$ : Efecto fijo asociado al j-esimo nivel del factor PB; $(\tau \gamma)_{i j}$ : Efecto debido a la interacción entre los dos factores; eijk: Efecto del error aleatorio para la $k$ - esima unidad experimental de la interacción $i j$ - esima entre los dos factores y $\delta_{i j k l}$ Efecto aleatorio de la $i$ - esima sub muestra asociada a la $k$ - esima unidad experimental de la interacción $i j$ - esima entre los dos factores.

Para que el modelo propuesto tuviera validez, sobre los residuales se verificaron los supuestos de normalidad (prueba de Shapiro-Wilk), homogeneidad de varianzas (prueba de Bartlett) y de independencia (mediante la prueba de DurbinWatson) (Montgomery, 2002). En el caso de la variable Rigidez Flexural, debido al incumplimiento de los supuestos con los datos originales, fue necesario aplicar la transformación logarítmica, verificando el cumplimiento de los supuestos.
Para evaluar el efecto de los tratamientos sobre las variables de respuesta, se utilizó la prueba de comparación múltiple de Tukey, la que determina cuáles de los tratamientos presentan diferencias significativas y cuáles son estadísticamente iguales. El procesamiento estadístico de la información obtenida, se realizó en el software de programación para el análisis de datos y gráficos de libre distribución R versión 2.11.1.

\section{RESULTADOS Y DISCUSIÓN}

Evaluación del efecto de la mezcla PLB: PB: La tabla 2 presenta los resultados de la Prueba de Comparación de Tukey para los diferentes tratamientos en la tensión de la PLB. Los resultados obtenidos muestran que, en el caso de la tensión, con un nivel de confianza de $5 \%$, no hubo diferencias significativas entre los tratamientos evaluados y, por tal razón, la incorporación de PB en las proporciones evaluadas, no incide en esta propiedad respecto del tratamiento control.

Tabla 2. Prueba de Comparación Múltiple de Tukey para la Tensión Media PLB.

\begin{tabular}{|c|c|c|c|c|c|}
\hline \multicolumn{2}{|c|}{ Niveles a comparar } & \multicolumn{2}{c|}{ Medias } & Diferencia & Valor-P \\
\hline $70: 30$ & $100: 0$ & 66,5 & 68,8 & $-2,32$ & 0,982 \\
\hline $80: 20$ & $100: 0$ & 73,1 & 68,8 & 4,23 & 0,906 \\
\hline $90: 10$ & $100: 0$ & 79,9 & 68,8 & 11,09 & 0,358 \\
\hline $80: 20$ & $70: 30$ & 73,1 & 66,5 & 6,55 & 0,734 \\
\hline $90: 10$ & $70: 30$ & 79,9 & 66,5 & 13,41 & 0,224 \\
\hline $90: 10$ & $80: 20$ & 79,9 & 73,1 & 6,87 & 0,706 \\
\hline
\end{tabular}

*Diferencia significativa a un nivel de $5 \%$.

Debido a que la mayor proporción de la fibra del PB es corta, que se compone por fibra corta de bagazo de caña, hasta un $80 \%$ y de fibra larga de resinosas, hasta un $20 \%$, se consideraba factible que al mezclarlo con el PLB, cuya fibra podría ser más homogénea o más larga, disminuyera la tensión de esta última; sin embargo, esta situación no se evidenció, posiblemente, por similitud en las características de las fibras mezcladas. Es posible que el uso de material reciclado para la elaboración de la PLB afecte la longitud de sus fibras, incidiendo en su tensión.

En el caso de la rigidez flexural (Tabla 3), todos los tratamientos con mezcla presentaron diferencias significativas, con un nivel de confianza del $5 \%$, respecto del tratamiento control. En todos los casos, la rigidez flexural de los tratamientos con mezcla fue mayor; lo cual, indicaría que la incorporación de PB aportó rigidez al PLB. Este comportamiento podría estar asociado con características físicas de la fibra larga del PB, como el espesor de la pared, que podría ser más grueso que el de la fibra del PLB y, por ende, aumentar su rigidez; sin embargo, esta hipótesis debe ser validada con pruebas de morfología de la fibra. En cuanto a la comparación de la rigidez flexural de las mezclas, no se encontraron diferencias significativas entre éstas.

Evaluación del efecto de la mezcla PLC: PB: La tabla 4 presenta los resultados de la prueba de comparación para los diferentes tratamientos en la tensión de la PLC. En el caso de la tensión, con un nivel de significancia de $5 \%$, solamente hubo diferencias significativas entre los tratamientos 70:30 y 100:00, siendo mayor en la primera, lo que indica que desde la perspectiva de esta variable, la primera mezcla fue la única de las evaluadas, que mejoró tensión respecto del tratamiento control.

La obtención de la PLC, a través de procesos mecánicos y el uso de pulpa reciclada para su fabricación, reducen tanto la longitud promedio de las fibras como la disponibilidad de los 
enlaces químicos -puentes de hidrógeno- entre sus fibras, generando una menor tensión respecto de pulpas obtenidas químicamente, las que además son más elásticas, por su menor contenido de lignina (Smook, 1990); aunque el
PB es obtenido químicamente tiene un máximo de $20 \%$ de fibra larga, lo que, posiblemente, hace necesario la adición de proporciones mayores o iguales que $30 \%$, para mejorar la tensión del blanco.

Tabla 3. Prueba de Comparación Múltiple de Tukey para la Rigidez Media PLB.

\begin{tabular}{|c|c|c|c|c|c|c|c|c|c|}
\hline \multicolumn{2}{|c|}{ Niveles a comparar } & \multicolumn{2}{|c|}{ Rigidez } & Media & Log-Rigidez & Media & Diferencia & Valor-P \\
\hline $70: 30$ & $100: 0$ & 2,66 & & 2,22 & 0,957 & & 0,78 & 0,177 & $0,034^{*}$ \\
\hline $80: 20$ & $100: 0$ & 2,84 & & 2,22 & 1,032 & & 0,78 & 0,251 & $0,001^{*}$ \\
\hline $90: 10$ & $100: 0$ & 2,91 & & 2,22 & 1,062 & & 0,78 & 0,282 & $<0,001^{*}$ \\
\hline $80: 20$ & $70: 30$ & 2,84 & & 2,66 & 1,032 & & 0,957 & 0,075 & 0,641 \\
\hline $90: 10$ & $70: 30$ & 2,91 & & 2,66 & 1,062 & & 0,957 & 0,105 & 0,352 \\
\hline $90: 10$ & $80: 20$ & 2,91 & & 2,84 & 1,062 & & 1,032 & 0,03 & 0,963 \\
\hline
\end{tabular}

*Diferencia significativa a un nivel de 5\%.

Tabla 4. Prueba de Comparación Múltiple de Tukey para la Tensión Media PLC.

\begin{tabular}{|c|c|c|c|c|c|}
\hline \multicolumn{2}{|c|}{ Niveles a comparar } & \multicolumn{2}{|c|}{ Medias } & \multirow{2}{*}{$\begin{array}{c}\text { Diferencia } \\
29,35\end{array}$} & \multirow{2}{*}{$\frac{\text { Valor-P }}{0,004 *}$} \\
\hline $70: 30$ & $100: 0$ & 84,7 & 55,4 & & \\
\hline $80: 20$ & $100: 0$ & 72,2 & 55,4 & 16,81 & 0,072 \\
\hline 90:10 & $100: 0$ & 68,4 & 55,4 & 13,01 & 0,182 \\
\hline $80: 20$ & $70: 30$ & 72,2 & 84,7 & $-12,54$ & 0,204 \\
\hline $90: 10$ & $70: 30$ & 68,4 & 84,7 & $-16,33$ & 0,081 \\
\hline $90: 10$ & $80: 20$ & 68,4 & 72,2 & $-3,79$ & 0,908 \\
\hline
\end{tabular}

*Diferencia significativa a un nivel de $5 \%$.

En la tabla 5, se presenta la prueba de comparación del ensayo de rigidez flexural para todos los tratamientos de PLC. Respecto de la rigidez flexural, se encontró que para un nivel de significancia de $5 \%$, el tratamiento control fue diferente a los otros tres evaluados. En todos los casos, la rigidez media del PLC sin mezclar fue mayor que la de las mezclas en porcentajes, que oscilan entre 26,7 y $45,6 \%$; de otro lado, no se encontraron diferencias significativas entre los tratamientos que incluían la mezcla del PLC con PB. El proceso de extracción de las fibras pudo ser el factor diferenciador, que incidió en este comportamiento.

Como se indicó anteriormente, la pulpa del PLC es producida mecánicamente y el PB químicamente; en el primer caso, la lignina, que impide la formación de puentes de hidrógeno, se conserva en la superficie de la fibra, haciéndola más rígida y, en el segundo, es disuelta, a través de los procesos de blanqueo químico, ejerciendo el efecto contrario, es decir, haciéndola más flexible. Como lo muestran los resultados, con todas las mezclas se logró disminuir la rigidez comparadas con el tratamiento control.

Finalmente, se concluye que la incorporación de papel bond al papel plegadiza blanco en las proporciones evaluadas, no ejerció efecto sobre la tensión, pero sí aumentó la rigidez flexural y no se encontraron diferencias en los valores de ambas propiedades físicas entre las mezclas. En caso de requerirse el aumento de la rigidez flexural del papel plegadiza blanco, la mejor proporción sería la mezcla PLB: PB 90:10.

El tratamiento papel plegadiza café y papel bond (PLC:PB) en una proporción 70:30 mostró diferencias significativas en las dos variables analizadas, comparado con el tratamiento control; con la mezcla, se obtuvo una pulpa con mayor tensión y más flexible, dado que incrementó el 52,9\%, en la tensión y descendió un 21,1\%, en la rigidez flexural, respec- 
tivamente. En caso de considerarse necesario conservar o aumentar la rigidez flexural, en la mezcla 70:30 podría aumentarse el gramaje, incrementando el volumen de pulpa, para formar la hoja.
Los tratamientos 90:10 y 80:20 solamente incidieron en la rigidez flexural, haciéndola menor que la del tratamiento control. Aunque la mezcla más indicada para una aplicación depende de las características del producto a obtener con la pulpa, el tratamiento 70:30 presenta la ventaja de influir en ambas propiedades físicas.

Tabla 5. Prueba de Comparación Múltiple de Tukey para la Rigidez Media PLC.

\begin{tabular}{|cc|c|l|l|l|c|c|}
\hline \multicolumn{2}{|c|}{$\begin{array}{c}\text { Niveles a } \\
\text { comparar }\end{array}$} & Rigidez & \multicolumn{1}{|c|}{ Media } & Log-Rigidez & \multicolumn{1}{c|}{ Media } & Diferencia & Valor-P \\
$70: 30$ & $100: 0$ & 2,62 & 3,32 & 0,945 & 1,182 & $-0,237$ & $0,007 *$ \\
\hline $80: 20$ & $100: 0$ & 2,37 & 3,32 & 0,849 & 1,182 & $-0,332$ & $<0,001 *$ \\
\hline $90: 10$ & $100: 0$ & 2,28 & 3,32 & 0,806 & 1,182 & $-0,376$ & $<0,001 *$ \\
\hline $80: 20$ & $70: 30$ & 2,37 & 2,62 & 0,849 & 0,945 & $-0,096$ & 0,522 \\
\hline $90: 10$ & $70: 30$ & 2,28 & 2,62 & 0,806 & 0,945 & $-0,14$ & 0,2 \\
\hline $90: 10$ & $80: 20$ & 2,28 & 2,37 & 0,806 & 0,849 & $-0,044$ & 0,923 \\
\hline
\end{tabular}

*Diferencia significativa a un nivel del $5 \%$

Los resultados muestran que la incorporación de papel bond al papel plegadiza, tanto blanco como café, incide en las propiedades físicas evaluadas, constituyendo una opción de valorización, dependiendo de los requerimientos de los productos finales. Esta opción de aprovechamiento evaluada tiene una alto potencial de aplicación en los contextos de las PMRS objeto de estudio.

Conflicto de intereses: El manuscrito fue preparado y revisado con la participación de todos los autores, quienes declaramos que no existe ningún conflicto de intereses que ponga en riesgo la validez de los resultados presentados.

\section{BIBLIOGRAFÍA}

1. AMERICAN SOCIETY FOR TESTING MATERIALS TECHNICAL ASSOCIATION OF THE PULP AND PAPER INDUSTRY 2001 -TAPPI-. Norma TAPPI T494 OM-2001. Tensile properties of paper and paperboard.

2. CASEY, J.P. 1991. Pulpa y papel: química y tecnología química. Vol. 3, Editorial Noriega- Limusa. 200p.

3. KUEHL, R.O. 2001. Diseño de Experimentos. Thomson Learning. Segunda edición. International Thomson Editores, S.A. México. 680p.
4. MARMOLEJO, L.F.; TORRES, P.; OVIEDO, É.R.; BEDOYA, D.F.; AMÉZQUITA, C.P.; KLINGER, R.; ALBÁN, F.; DÍAZ, L.F. 2009. Flujo de residuos: Elemento base para la sostenibilidad del aprovechamiento de residuos sólidos municipales. Rev. Ing. Competitiv. 11:79-93.

5. MERRILD, H.; DAMGAARD, A.; CHRISTENSEN, T. 2008. Life cycle assessment of waste paper management: The importance of technology data and system boundaries in assessing recycling and incineration. Res. Conserv. Recyc. 52:1391-1398.

6. MINISTERIO DE AMBIENTE, VIVIENDA Y DESARROLLO TERRITORIAL -MAVDT- \& ESTUDIOS Y PROYECTOS AMBIENTALES Y MECÁNICOS -EPAM S.A. E.S.P.-. 2008 Construcción de criterios técnicos para el aprovechamiento y valorización de residuos sólidos orgánicos con alta tasa de biodegradación, plásticos, vidrio, papel y cartón. . Manual 3: Orgánicos, papel y cartón. Bogotá D.C. 91p.

7. MONTGOMERY, D. 2002. Diseño y Análisis de Experimentos. $2^{\mathrm{a}}$ Edición. México D.F.: Limusa Wiley. $686 p$.

8. PEDRAZA, M.L.; COSSIO, L.P.; HILARIO, A.C. 2007. Aprovechamiento de Papel reciclado. Transferencias 
de tecnologías limpias en el sector de residuos sólidos. Lima, CONCYTEC - OEA.

9. SMOOK, G.A. 1990. Manual para técnicos de pulpa y papel. $4^{\text {a }}$ Ed. Atlanta: TAPPI Press. 396p.

10. SUPERINTENDENCIA DE SERVICIOS PÚBLICOS DOMICILIARIOS -SSPD-. 2008. Diagnóstico sectorial: plantas de aprovechamiento de residuos sólidos. Dis- ponible desde internet en http://www.superservicios. gov.co/c/document_library/get file?uuid=73cfd722c46e-4caf-b03f-486810f6536 c \& groupld $=10122$ (con acceso 02/12/2012).

Recibido: Agosto 6 de 2013

Aceptado: Marzo 28 de 2014

Como citar:

Gutiérrez - R., J.A.; Marmolejo - Rebellón, L.F.; Albán Achinte, F.; Torres, W.A.; Torres - Lozada, P. 2014. Mejoramiento de propiedades mecánicas del papel plegadiza recuperado de residuos sólidos municipales. Rev. U.D.C.A Act. \& Div. Cient. 17(1): 237-243. 
